Ferrata Storti Foundation

\title{
DA-EPOCH-R combined with high-dose methotrexate in patients with newly diagnosed stage II-IV CD5-positive diffuse large B-cell lymphoma: a single-arm, open-label, phase II study
}

Haematologica 2020

Volume 105(9):2308-2315

\section{Correspondence:}

KANA MIYAZAKI

kmiyazaki@clin.medic.mie-u.ac.jp

Received: July 11, 2019.

Accepted: October 21, 2019.

Pre-published: October 24, 2019.

doi:10.3324/haematol.2019.231076

(C)2020 Ferrata Storti Foundation

Material published in Haematologica is covered by copyright. All rights are reserved to the Ferrata Storti Foundation. Use of published material is allowed under the following terms and conditions:

https://creativecommons.org/licenses/by-nc/4.0/legalcode. Copies of published material are allowed for personal or internal use. Sharing published material for non-commercial purposes is subject to the following conditions:

https://creativecommons.org/licenses/by-nc/4.0/legalcode, sect. 3. Reproducing and sharing published material for commercial purposes is not allowed without permission in writing from the publisher.

\begin{abstract}
Kana Miyazaki, ${ }^{1}$ Naoko Asano, ${ }^{2}$ Tomomi Yamada, ${ }^{3}$ Kohta Miyawaki, ${ }^{4}$ Rika Sakai, ${ }^{5}$ Tadahiko Igarashi, ${ }^{6}$ Momoko Nishikori, ${ }^{7}$ Kinya Ohata, ${ }^{8}$ Kazutaka Sunami, ${ }^{9}$ Isao Yoshida, ${ }^{10}$ Go Yamamoto, ${ }^{11}$ Naoki Takahashi, ${ }^{12}$ Masataka Okamoto, ${ }^{13}$ Hiroki Yano,${ }^{14}$ Yuki Nishimura,,${ }^{15}$ Satoshi Tamaru, ${ }^{15}$ Masakatsu Nishikawa, ${ }^{15}$ Koji Izutsu, ${ }^{11,16}$ Tomohiro Kinoshita, ${ }^{17}$ Junji Suzumiya, ${ }^{18}$ Koichi Ohshima, ${ }^{19}$ Koji Kato, ${ }^{4}$ Naoyuki Katayama ${ }^{1}$ and Motoko Yamaguchi ${ }^{1}$
\end{abstract}

${ }^{1}$ Department of Hematology and Oncology, Mie University Graduate School of Medicine, Mie; ${ }^{2}$ Department of Molecular Diagnostics, Nagano Prefectural Shinshu Medical Center, Suzaka; ${ }^{3}$ Department of Medical Innovation, Osaka University Hospital, Suita; ${ }^{4}$ Department of Medicine and Biosystemic Science, Kyushu University Graduate School of Medical Sciences, Fukuoka; ${ }^{5}$ Department of Medical Oncology, Kanagawa Cancer Center, Yokohama; ${ }^{6} \mathrm{Hematology/Oncology} \mathrm{Division,} \mathrm{Gunma} \mathrm{Cancer} \mathrm{Center,} \mathrm{Ohta;}$ ${ }^{7}$ Department of Hematology and Oncology, Kyoto University Graduate School of Medicine, Kyoto; ${ }^{8}$ Department of Hematology, Kanazawa University, Kanazawa; ${ }^{9}$ Department of Hematology, National Hospital Organization Okayama Medical Center, Okayama; ${ }^{10}$ Department of Hematologic Oncology, National Hospital Organization Shikoku Cancer Center, Matsuyama; ${ }^{11}$ Department of Hematology, Toranomon Hospital, Tokyo; ${ }^{12}$ Department of Hematology and Oncology, International Medical Center, Saitama Medical University, Hidaka; ${ }^{13}$ Department of Hematology, Fujita Health University School of Medicine, Toyoake; ${ }^{14}$ Department of Hematology, Kainan Hospital Aichi Prefectural Welfare Federation of Agricultural Cooperatives, Yatomi; ${ }^{15}$ Clinical Research Support Center, Mie University Hospital, Tsu; ${ }^{16}$ Department of Hematology, National Cancer Center Hospital, Tokyo; ${ }^{17}$ Department of Hematology and Cell Therapy, Aichi Cancer Center Hospital, Nagoya; ${ }^{18}$ Department of Oncology and Hematology, Shimane University Hospital, Izumo and ${ }^{19}$ Department of Pathology, Kurume University School of Medicine, Kurume, Japan

\section{ABSTRACT}

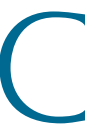
D5-positive diffuse large B-cell lymphoma (CD5+ DLBCL) is characterized by poor prognosis and a high frequency of central nervous system relapse after standard immunochemotherapy. We conducted a phase II study to investigate the efficacy and safety of dose-adjusted (DA)EPOCH-R (etoposide, prednisone, vincristine, cyclophosphamide, doxorubicin, and rituximab) combined with high-dose methotrexate (HD-MTX) in newly diagnosed patients with $\mathrm{CD} 5^{+} \mathrm{DLBCL}$. Previously untreated patients with stage II to IV CD5 ${ }^{+}$DLBCL according to the 2008 World Health Organization classification were eligible. Four cycles of DA-EPOCH-R followed by two cycles of HD-MTX and four additional cycles of DAEPOCH-R (DA-EPOCH-R/HD-MTX) were planned as the protocol treatment. The primary end point was 2-year progression-free survival (PFS). Between September 25, 2012, and November 11, 2015, we enrolled 47 evaluable patients. Forty-five (96\%) patients completed the protocol treatment. There were no deviations or violations in the DA-EPOCH-R dose levels. The complete response rate was $91 \%$, and the overall response rate was $94 \%$. At a median follow up of 3.1 years (range, 2.0-4.9 years), the 2year PFS was 79\% [95\% confidence interval (CI): 64-88]. The 2-year overall survival was $89 \%$ (95\% CI: 76-95). Toxicity included grade 4 neutropenia in $46(98 \%)$ patients, grade 4 thrombocytopenia $12(26 \%)$ patients, and febrile neutropenia in $31(66 \%)$ patients. No treatment-related death was noted during the study. DA-EPOCH-R/HD-MTX might be a first-line therapy option for stage II-IV CD5 ${ }^{+}$DLBCL and warrants further investigation. (Trial registered at: UMIN-CTR: UMINO00008507.) 


\section{Introduction}

Diffuse large B-cell lymphoma (DLBCL) includes a heterogeneous group of lymphomas with various clinicopathological and genetic features. ${ }^{1,2}$ The current standard first-line therapy of DLBCL is rituximab, cyclophosphamide, doxorubicin, vincristine, and prednisone (R-CHOP). Gene expression profiling (GEP) has identified activated B-cell-like $(A B C)$, germinal center B-cell-like (GCB), and unclassified DLBCL. ${ }^{3}$ ABC DLBCL exhibits worse prognosis than GCB DLBCL after R-CHOP. ${ }^{4}$

CD5-positive $\left(\mathrm{CD}^{+}\right)$DLBCL is an immunohistochemical subgroup of DLBCL, not otherwise specified (NOS) in the 2008 World Health Organization (WHO) classification and accounts for $5-10 \%$ of DLBCL. ${ }^{1}$ It is associated with various clinical characteristics, such as elderly onset, advanced stage at diagnosis, elevated lactate dehydrogenase (LDH) level, and frequent involvement of extranodal sites. ${ }^{5-10}$ Ninety percent of $\mathrm{CD}^{+}$DLBCL cases are positive for BCL2, ${ }^{6,9}$ which has been reported as a risk factor for short survival in DLBCL. The patients with $\mathrm{CD}^{+} \mathrm{DLBCL}$ show significantly worse survival than those with CD5 negative (CD5-) DLBCL after R-CHOP, ${ }^{7,-14}$ and is also characterized by a high incidence of central nervous system (CNS) relapse $(8-13 \%)$, mainly brain parenchymal relapse.$^{8,10} \mathrm{CNS}$ relapse strongly affects the prognosis of CD5 $5^{+}$DLBL. ${ }^{8}$ Up to $80 \%$ of $C D 5^{+}$DLBCL are classified as $A B C$ DLBCL by GEP, ${ }^{9,15,16}$ and CD5 expression is significantly associated with the poor prognosis of patients with ABC DLBCL., ${ }^{9,15}$

Dose-adjusted etoposide, prednisone, vincristine, cyclophosphamide, doxorubicin, and rituximab (DAEPOCH-R) is an infusional regimen that incorporates a dynamic dose adjustment and has shown excellent efficacy in BCL2 ${ }^{+}$DLBCL. ${ }^{17,18}$ High-dose methotrexate (HDMTX) is commonly used in the treatment of primary DLBCL of the CNS. ${ }^{19}$ To explore a more effective first-line chemotherapy for $\mathrm{CD}^{+} \mathrm{DLBCL}$, we have been investigating DA-EPOCH-R combined with high-dose methotrexate (HD-MTX) therapy (DA-EPOCH-R/HD-MTX) since $2009 .{ }^{20}$ In 2009, when we were planning this study, eight cycles of R-CHOP was used as the standard treatment for the patients with advanced DLBCL. ${ }^{21}$ In previous phase II trials using DA-EPOCH-R, patients received two cycles beyond complete response (CR) or stable changes after six cycles of DA-EPOCH-R. ${ }^{17,18}$ Considering the aggressiveness of $\mathrm{CD}^{+}$DLBCL, we selected eight cycles of DAEPOCH-R based on the maximum number of cycles in original studies of DA-EPOCH-R..$^{17,18,22}$ Because most CNS relapse events in patients with $\mathrm{CD} 5^{+} \mathrm{DLBCL}$ during R-CHOP have been documented within 6 months after diagnosis, ${ }^{8}$ we added two cycles of HD-MTX between the fourth and the fifth cycles of DA-EPOCH-R. In our retrospective analysis of five patients with untreated stage IV $\mathrm{CD}^{+}$DLBCL who received DA-EPOCH-R/HD-MTX, the overall response rate (ORR) was 100\%, and all toxicities were manageable. ${ }^{20}$ Of note, all of the patients belonged to the high-risk group of the International Prognostic Index (IPI) ${ }^{23}$ and three patients had CNS involvement at diagnosis. To further evaluate the efficacy of DA-EPOCH-R/HDMTX for newly diagnosed CD5 $5^{+}$DLBCL, we have been conducting a prospective, multicenter, single-arm, openlabel, phase II trial in 31 hospitals in Japan since 2015 (registered at: UMIN000008507). Here, we report the primary analysis results of that trial.

\section{Methods}

\section{Study design and participants}

In this multicenter phase II trial, eligible patients were 20-75 years of age and had newly diagnosed $\mathrm{CD} 5^{+} \mathrm{CD} 20^{+}$DLBCL with Ann Arbor stage II-IV disease (see Online Supplementary Methods). The diagnosis of $\mathrm{CD}^{+}$DLBCL was made according to the 2008 WHO criteria ${ }^{1}$ as DLBCL, NOS with CD5 and CD20 expression in tumor cells based on immunohistochemistry and/or flow cytometry. A complete list of inclusion and exclusion criteria is shown in Online Supplementary Table S1. The study was approved by the protocol review committee of the study as well as the institutional review board of each institution in accordance with the Declaration of Helsinki. Written informed consent was obtained from all patients before enrollment.

\section{Treatment}

The protocol treatment comprised four cycles of DA-EPOCH-R followed by two cycles of HD-MTX and four additional cycles of DA-EPOCH-R. DA-EPOCH-R was administered according to original reports. ${ }^{17,18,22}$ The treatment details are described in the Online Supplementary Methods. The fourth and fifth cycles of DAEPOCH-R were administered at the same dose level. MTX was administered intravenously at a dose of $3.5 \mathrm{~g} / \mathrm{m}^{2}$ on a 14-day cycle with leucovorin rescue. The patients did not receive intrathecal chemotherapy. Acyclovir and trimethoprim-sulfamethoxazole were administered prophylactically. Radiation therapy was not permitted during the protocol treatment.

\section{Diffuse large B-cell lymphoma subtyping}

Following patient enrollment, formalin-fixed paraffin-embedded (FFPE) sections were histologically reviewed according to both the 2008 WHO criteria and the 2017 WHO criteria by the central pathology review board because the WHO classification was updated during the study period. ${ }^{1,2}$ DLBCL cell-of-origin (COO) subtypes were determined based on an immunohistochemical method developed by Hans et al. ${ }^{24}$ and gene-expression-based Lymph2Cx assay. ${ }^{25}$ The details of immunohistochemistry and the methods of Lymph2Cx assay are given in the Online Supplementary Methods.

\section{End points}

The primary end point was 2-year progression-free survival (PFS) (see the Online Supplementary Methods). Secondary end points were CR rate, ORR, 2-year overall survival (OS), 2-year CNS relapse rate, and toxicity. As a preplanned exploratory analysis, we assessed survival in each subgroup of COO, IPI, and CNS-IPI categories. ${ }^{26}$ Treatment responses were assessed at each participating institute using positron emission tomography-computed tomography within 6-8 weeks after the beginning of the eighth cycle of DA-EPOCH-R according to the revised International Working Group Criteria. ${ }^{27}$ Toxicity was graded according to the Common Terminology Criteria for Adverse Events version 4.0.

\section{Statistical analysis}

Because this was the first clinical trial for untreated $\mathrm{CD} 5^{+}$ DLBCL, we obtained historical controls of 2-year PFS (51\%) from the data set of our previous retrospective study of $\mathrm{CD} 5^{+}$DLBCL. ${ }^{8}$ (See the Online Supplementary Methods and Online Supplementary Figure S1 for details) We anticipated that 41 evaluable patients were needed to achieve $80 \%$ power to detect a $20 \%$ difference in 2-year PFS from the historical control (51\%) with a one-sided type I error of 0.05 . The planned sample size was 45 patients with the expectation that $10 \%$ would be ineligible. Survival estimates were calculated using the Kaplan-Meier method, and 95\% confidence 


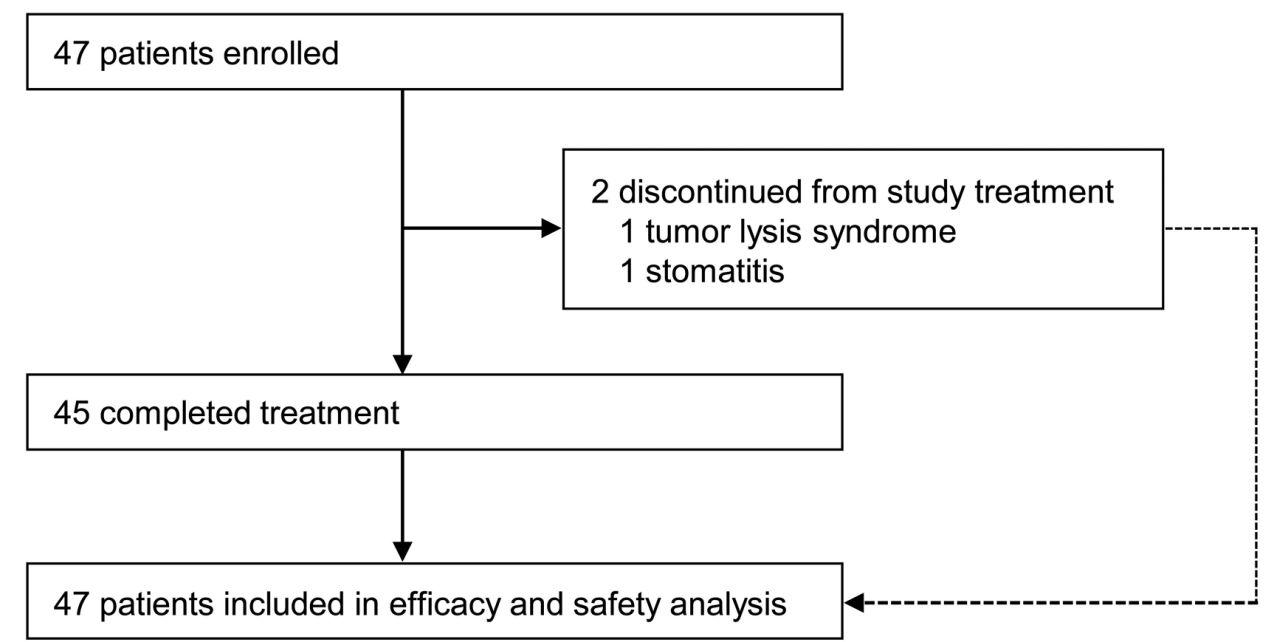

Figure 1. Trial profile.

intervals (CI) were estimated using Greenwood's formula. All analyses were performed using SAS v9.4 (Cary, NC, USA) statistical package.

\section{Results}

\section{Patients' characteristics}

A total of 47 patients from 24 participating institutions were enrolled in the study between July 2012 and November 2015 (Figure 1). The central pathology review confirmed the diagnosis of all patients as $\mathrm{CD} 5^{+} \mathrm{DLBCL}$ based on the 2008 WHO criteria. The review also revealed that two patients had high-grade B-cell lymphoma, NOS based on the 2017 WHO classification. The baseline clinical features and disease characteristics are listed in Table 1. Thirty-six percent of the patients were over 65 years of age. Skin/subcutaneous tissue was the most frequent site of extranodal involvement $(15 \%)$. One patient had primary testicular DLBCL.

For one case, the quality of the RNA extracted from the FFPE tissue sample was inadequate, but the remaining 46 cases were analyzed using GEP. Thirty-nine (85\%) of 46 cases were classified as ABC DLBCL, four (9\%) were classified as GCB DLBCL, and three (7\%) were unclassified. The morphological and immunophenotypical features are summarized in Table 2. Based on Hans' criteria, $72 \%$ of the 46 patients examined were classified as non-GCB DLBCL.

\section{Treatment and response}

Forty-five $(96 \%)$ patients completed the protocol treatment. In the remaining two patients, treatment was discontinued because of tumor lysis syndrome (TLS) with hyperkalemia after the first administration of rituximab and grade 3 stomatitis during the sixth cycle of DAEPOCH-R. The dose-adjustment map of DA-EPOCH-R is shown in Online Supplementary Figure S2. The range of the dose level of DA-EPOCH-R was 1-4; median dose level of DA-EPOCH-R was two. The maximum dose level of DAEPOCH-R was 1 in $26.1 \%, 2$ in $32.6 \%, 3$ in $26.1 \%, 4$ in $15.2 \%$, and $>4$ in $0 \%$. There were no deviations or viola- tions in determining the dose levels of DA-EPOCH-R. All patients were administered with DA-EPOCH-R/HDMTX entirely in the hospital. Of the 47 patients evaluable for response, 43 patients achieved a CR (91\%; 95\% CI: $80-$ $98 \%$ ), one patient achieved a partial response, and two patients experienced progressive disease. The ORR was 94\% (95\% CI: 82-99\%).

\section{Survival}

With a median follow up of 3.1 years (range, 2.0-4.9), the 2 -year PFS was $79 \%$ (90\% CI: $67-87 \%$; 95\%CI: $64-$ $88 \%$ ) (Figure 2A). This finding compared favorably with that of the historical control (51\%). The 2-year OS was 89\% (90\% CI: 79-95\%; 95\% CI: 76-95\%) (Figure 2B). One patient with CR died in a traffic accident 0.8 years after enrollment.

In a preplanned exploratory subgroup analysis, there was no significant difference in PFS (Online Supplementary Figure S3A) or OS (Online Supplementary Figure S3B) according to $\mathrm{COO}$ categories. The 2 -year PFS and OS for $\mathrm{CD}^{+} \mathrm{ABC}$ DLBCL $(\mathrm{n}=39$ ) were $77 \%$ and $87 \%$, respectively. There was no significant difference in PFS according to gender and each risk factor for IPI (data not shown).

\section{Central nervous system relapse}

The 2-year CNS relapse rate was 9\% (n=4; 90\%CI: 419\%, 95\% CI: 3-21\%) (Figure 3A). During the follow-up period, no patient experienced CNS relapse more than 2 years from enrollment. There was no significant difference in PFS among the CNS-IPI categories $(P=0.58)$ (Figure $3 \mathrm{~B})$. Ten patients in the CNS IPI high-risk group were classified as $A B C(n=8), \operatorname{GCB}(n=1)$, and unclassified DLBCL $(n=1)$.

The clinical characteristics of four patients who experienced CNS relapse are summarized in Online Supplementary Table S2. Among them, one patient had primary testicular DLBCL. Two patients were diagnosed as having high-grade B-cell lymphoma, NOS with MYC rearrangement based on the 2017 WHO criteria. Neither of these patients had the BCL2 translocation, and both were positive for CD10 and BCL2 by immunohistochemistry. For the remaining one patient, the protocol treat- 
Table 1. Baseline clinical features and disease characteristics.

\begin{tabular}{lc} 
Characteristic & All patients $(\mathbf{n}=\mathbf{4 7})$ \\
Age, years & \\
Median & 62 \\
Range & $37-74$ \\
$>60$ years & $28(60 \%)$ \\
Sex & \\
Male & $18(38 \%)$ \\
Female & $29(62 \%)$ \\
Stage & \\
II & $20(43 \%)$ \\
III-IV & $27(57 \%)$ \\
ECOG PS & \\
0 or 1 & $45(96 \%)$ \\
$>1$ & $2(4 \%)$ \\
\hline
\end{tabular}

\section{Serum LDH level}

\begin{tabular}{ll} 
Normal & $16(34 \%)$ \\
Elevated & $31(66 \%)$ \\
Extranodal sites & \\
0 or 1 & $31(66 \%)$ \\
$>1$ & $16(34 \%)$ \\
\hline
\end{tabular}

IPI risk category

Low/Low-intermediate $\quad 25(53 \%)$

High-intermediate/High

$22(47 \%)$

CNS-IPI risk category

$\begin{array}{ll}\text { Low } & 14(30 \%) \\ \text { Intermediate } & 23(49 \%) \\ \text { High } & 10(21 \%)\end{array}$

Cell-of-origin*

\begin{tabular}{lc}
$\mathrm{ABC}$ & $39(85 \%)$ \\
$\mathrm{GCB}$ & $4(9 \%)$ \\
Unclassified & $3(7 \%)$ \\
\hline
\end{tabular}

ABC: activated B-cell-like; CNS: central nervous system; EBER: Epstein-Barr virus-encoded RNA-1. GCB: germinal center-B-cell-like; IPI: International Prognostic Index; PS: performance status. *Examined by Lymph2Cx using formalin-fixed and paraffin-embedded tissues $(n=46)$.

ment was discontinued after the first drug administration due to grade 4 TLS. CNS relapses occurred in two patients (one patient had leptomeningeal disease with tumor of ethmoid sinus, and the other patient had brain parenchymal disease alone) before HD-MTX of the protocol treatment.

\section{Toxicity}

Toxicity was assessed in all 445 cycles of DA-EPOCHR/HD-MTX with 357 cycles of DA-EPOCH-R in all 47 patients. Table 3 lists adverse events in all 445 cycles of DA-EPOCH-R/HD-MTX, and Online Supplementary Table S3 lists adverse events in 357 cycles of DA-EPOCH-R. There were no treatment-related deaths. The most common grade 3 non-hematologic toxicity was elevated ALT $(28 \%)$. Four patients experienced grade 3 infection, including catheter-related infection, cellulitis, endocarditis, and urinary tract infection. Grade 3 peripheral motor neuropathy and peripheral sensory neuropathy occurred in $4 \%$ of patients, separately. There was no grade 3 cardiac toxicity. Grade 4 neutropenia and thrombocytopenia were present in $98 \%$ and $26 \%$ of patients, respectively.

The targeted ANC $\left(<0.5 \times 10^{9} / \mathrm{L}\right)$ occurred in $77 \%$ of
Table 2. Morphological and immunophenotypical features.

\begin{tabular}{lc} 
Morphology & All patients (n=47) \\
Common variant & $31(66 \%)$ \\
Giant cell rich variant & $3(6 \%)$ \\
Polymorphic variant & $12(26 \%)$ \\
Immunoblastic variant & $1(2 \%)$ \\
Immunohistochemistry & \\
CD5 $^{*}$ & $47(100 \%)$ \\
CD20 $^{-1}$ & $47(100 \%)$ \\
CyclinD1 & $0(0 \%)$ \\
CD10 $^{\dagger}$ & $13(28 \%)$ \\
BCL6 $^{\dagger}$ & $39(85 \%)$ \\
MUM1 $^{\dagger}$ & $44(96 \%)$ \\
MYC $^{\dagger}$ & $26(57 \%)$ \\
BCL2 $^{\dagger}$ & $45(98 \%)$ \\
MYC positive/BCL2 positive $^{\dagger}$ & $26(57 \%)$ \\
COO subtype by Hans' criteria $^{\dagger}$ & \\
GCB $^{\dagger}$ & $13(28 \%)$ \\
Non-GCB $^{\dagger}$ & $33(72 \%)$ \\
EBER $^{\dagger}$ sit situ & $0(0 \%)$ \\
\hline
\end{tabular}

COO: cell-of-origin; EBER: Epstein-Barr virus-encoded RNA-1; GCB: germinal center-Bcell-like. *Assessed by flow cytometry in 4 patients. 'Examined in 46 patients.

cycles. Thrombocytopenia of $<25 \times 10^{9} / \mathrm{L}$ occurred in $8 \%$ of cycles. Febrile neutropenia (FN) occurred in $66 \%$ of patients and 23\% per cycle of DA-EPOCH-R (Table 3 and Online Supplementary Table S3). All patients who experienced FN recovered from the toxicity soon after initial antibiotics therapy. Possible second malignancies were documented in three patients (age, 64-74 years; acute myeloid leukemia, $\mathrm{n}=1$; glioblastoma, $\mathrm{n}=1$; and colon cancer in adenoma, $\mathrm{n}=1$ ). In two of these patients, the second malignancy was diagnosed after salvage chemotherapy for relapsed $\mathrm{CD}^{+} \mathrm{DLBCL}$.

\section{Discussion}

To the best of our knowledge, this is the first clinical trial for $\mathrm{CD}^{+}$DLBCL. At the beginning of this trial in 2015, we calculated the threshold 2-year PFS using our previous retrospective study including 337 patients with $\mathrm{CD}^{+}$DLBCL. In 149 patients with newly diagnosed stage II-IV CD5 ${ }^{+}$DLBCL who received rituximab-containing chemotherapy (R-chemo) and had available follow-up data, the 2-year PFS was $51 \%$. Recently, the results of several retrospective studies of $\mathrm{CD}^{+}$DLBCL in the rituximab-era (R-era) were reported. In both cohorts of 102 patients with all stage $\mathrm{CD} 5^{+}$DLBCL who received R-chemo at nine institutes in the United States ${ }^{10}$ and 31 patients with all stage $\mathrm{CD}^{+}$DLBCL who received RCHOP in other Western countries, ${ }^{9}$ the 2-year OS was less than $50 \%$. Therefore, we consider that our threshold setting was reasonable and that the 2-year PFS (79\%) by the protocol treatment in our present study was superior to the historical control.

Few reports have described the use of DA-EPOCH-R for $\mathrm{CD}^{+}$DLBCL. For all DLBCL subtypes, there was no sig- 
nificant difference in survival between patients who received R-CHOP and patients who received DA-EPOCH$\mathrm{R}$ in a large randomized controlled trial. ${ }^{28}$ In that trial, the prognosis stratified by CD5 was not shown. In the largest study including 130 DLBCL patients who were examined for CD5 expression and received DA-EPOCH-R, 16 $(12.3 \%)$ patients had $\mathrm{CD}^{+}$DLBCL. ${ }^{29}$ At a median follow up of 28.5 months, $37.5 \%$ of patients in the $C D 5^{+}$DLBCL group had died, which showed a significantly worse survival than those in the CD5- DLBCL. The 2-year OS was $89 \%$ (90\%CI: $79-95 \%$; 95\% CI: 76-95\%) for DA-EPOCH$\mathrm{R} / \mathrm{HD}-\mathrm{MTX}$ in our present trial.

In a large retrospective study including 102 patients with $\mathrm{CD}^{+}$DLBCL, 6 of 7 patients with untreated $\mathrm{CD}^{+}$ DLBCL who received DA-EPOCH-R achieved a CR and were disease-free with a median follow up of 30 months. ${ }^{10}$ In another study, CD5 expression in DLBCL was identified as a risk factor for short OS in a cohort of patients who received $\mathrm{R}-\mathrm{EPOCH},{ }^{29}$ which indicated that $\mathrm{R}-\mathrm{EPOCH}$ alone may be insufficient to cure this disease. In the present study of stage II-IV CD5 ${ }^{+}$DLBCL, HD-MTX therapy was added between the fourth and fifth cycles of DAEPOCH-R. In our trial, the completion rate of the protocol treatment was high. Moreover, there were no deviations or violations in the dose-adjustment of DA-EPOCH-R. All patients received DA-EPOCH-R/HD-MTX only in the hospital. These features may have contributed to the excellent efficacy of DA-EPOCH-R/HD-MTX in our trial. However, a considerable number of female patients $(62 \%)$, patients with PS $<2(96 \%)$, and those with
A

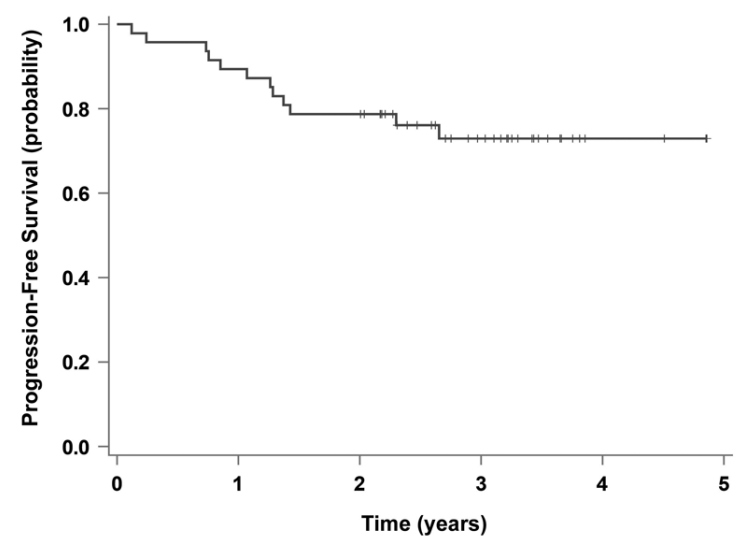

B

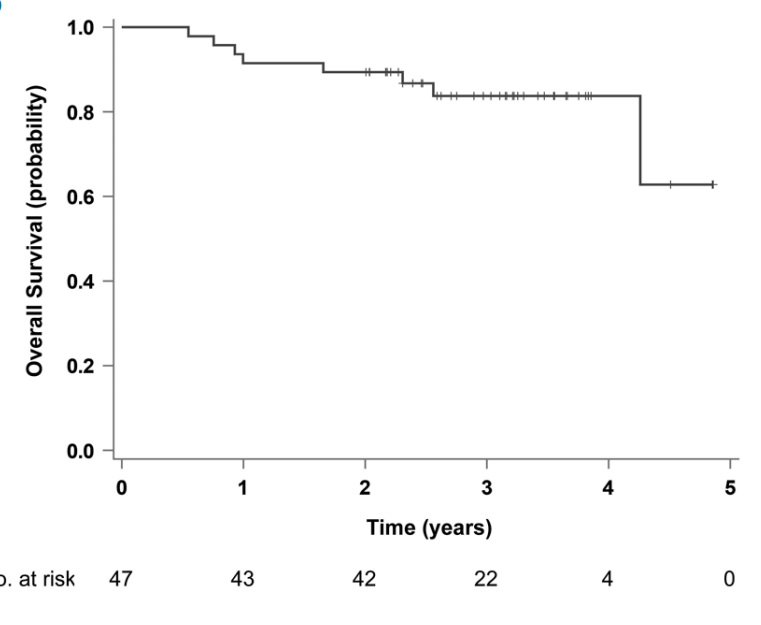

Figure 2. Survival curves for patients with CD5-positive diffuse large B-cell lymphoma. (A) Progression-free survival and (B) overall survival for all patients ( $\mathrm{n}=47$ ).

A

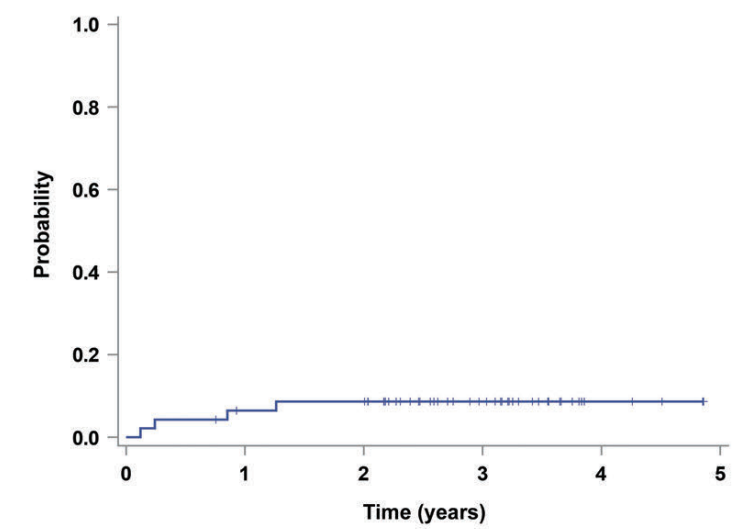

B

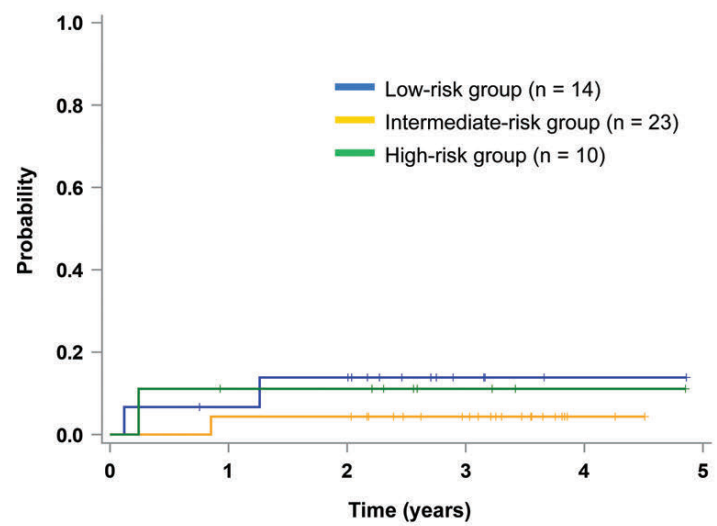

\section{No. at risk}

$\begin{array}{lcccccc}\text { Low } & 15 & 12 & 11 & 3 & 1 & 0 \\ \text { Intermediate } & 23 & 22 & 22 & 15 & 2 & 0 \\ \text { High } & 10 & 8 & 8 & 4 & 1 & 0\end{array}$

Figure 3. Central nervous system (CNS) relapse in CD5-positive diffuse large B-cell lymphoma patients ( $n=47$ ). (A) Probability of CNS relapse. (B) Probability of CNS relapse according to the CNS-International Prognostic Index. 
low/low-intermediate IPI risk (53\%) were enrolled in the present study. In the subgroup analysis of PFS, there was no significant difference between those with and those without risk factors for gender, PS, and IPI group, although the sample size was small. Considering these results, further studies are needed to establish DA-EPOCH-R/HDMTX as a standard therapy for newly diagnosed stage IIIV CD5 $5^{+}$DLBCL. Nevertheless, we consider that this regimen might be regarded as a treatment option, because CD5 expression can be easily examined in clinical practice.

The incidence of CNS relapse for all patients with DLBCL treated with R-CHOP has been reported to be 1.9$6.4 \%{ }^{30-32}$ whereas the incidence of CNS relapse for $\mathrm{CD}^{+}$ DLBCL in the R-era were reported as $13 \%$ in our retrospective study, ${ }^{8} 8 \%$ in a study in Western countries, ${ }^{9}$ and $33 \%$ in ten patients treated with DA-EPOCH-R in a retrospective study. ${ }^{29}$ Although our present trial does not have a sufficient statistical power to assess the reduction in CNS relapse, the incidence of CNS relapse in the present study (9\%; 95\%CI: 3-21\%) seems to be lower than that for DA-EPOCH-R alone. To further reduce the incidence of CNS relapse, development of systemic chemotherapy in combination with one or more newer agents that cross the blood-brain barrier, including ibrutinib, ${ }^{33,34}$ lenalidomide ${ }^{35}$ and tumor necrosis factor- $\alpha$ coupled with $\mathrm{NGR}^{36}$ is warranted. Combining a high CNS-IPI score and $\mathrm{ABC} /$ classified $\mathrm{COO}$ were reported to identify a patient subgroup at high risk for developing CNS relapse. ${ }^{37}$ Of note, in the present trial, $90 \%$ of patients in the CNS-IPI high-risk group were $\mathrm{ABC}$ /unclassified DLBCL. However, in four patients with CNS relapse, one patient had primary testicular lymphoma, which should be treated with a specific treatment strategy including local radiotherapy. ${ }^{38}$ Moreover, 2 of the 4 patients with CNS relapse had highgrade B-cell lymphoma, NOS with MYC translocation, which is known to result in a relatively high incidence of CNS relapse. ${ }^{39}$ These results suggest that $\mathrm{CD}^{+}$DLBCL diagnosed according to the 2008 WHO criteria was a heterogeneous lymphoma and that DA-EPOCH-R/HD-MTX might be beneficial for patients with $\mathrm{CD} 5^{+} \mathrm{DLBCL}$ in the WHO 2017 era.

As expected, the incidence of grade 3 peripheral motor neuropathy, peripheral sensory neuropathy and cardiac events was low $(4 \%, 4 \%$, and $0 \%$, respectively) and was comparable to that in previous studies including DAEPOCH-R. ${ }^{17,18}$ Two-thirds $(66 \%)$ of the patients in this study experienced FN, and the incidence was higher than

Table 3. Hematologic and non-hematologic toxicities.

\begin{tabular}{|c|c|c|c|c|}
\hline & Grade 1-2 & Grade 3 & Grade 4 & Grade 5 \\
\hline \multicolumn{5}{|l|}{ Hematologic adverse event } \\
\hline Neutropenia & 0 & $1(2 \%)$ & $46(98 \%)$ & 0 \\
\hline Leukopenia & $1(2 \%)$ & 0 & $46(98 \%)$ & 0 \\
\hline Thrombocytopenia & $13(28 \%)$ & $22(47 \%)$ & $12(26 \%)$ & 0 \\
\hline Anemia & $11(23 \%)$ & $26(55 \%)$ & $10(21 \%)$ & 0 \\
\hline Febrile neutropenia & - & $31(66 \%) *$ & 0 & 0 \\
\hline \multicolumn{5}{|l|}{ Non-hematologic adverse event } \\
\hline Blood bilirubin increased & $9(19 \%)$ & $2(4 \%)$ & 0 & 0 \\
\hline AST increased & $36(77 \%)$ & $5(11 \%)$ & 0 & 0 \\
\hline ALT increased & $30(64 \%)$ & $13(28 \%)$ & 0 & 0 \\
\hline Hyperglycemia & $40(85 \%)$ & $1(2 \%)$ & 0 & 0 \\
\hline Hyponatremia & $36(77 \%)$ & $7(15 \%)$ & 0 & 0 \\
\hline Hyperkalemia & $20(43 \%)$ & 0 & $1(2 \%)^{\dagger}$ & 0 \\
\hline Hypokalemia & $38(81 \%)$ & $8(17 \%)$ & 0 & 0 \\
\hline Hypocalcaemia & $44(94 \%)$ & $1(2 \%)$ & $1(2 \%)$ & 0 \\
\hline Cardiac disorders & - & 0 & 0 & 0 \\
\hline Constipation & $31(66 \%)$ & $3(6 \%)$ & 0 & 0 \\
\hline Ileus & $1(2 \%)$ & $2(4 \%)$ & 0 & 0 \\
\hline Nausea & $33(70 \%)$ & $1(2 \%)$ & 0 & 0 \\
\hline Vomiting & $9(19 \%)$ & $1(2 \%)$ & 0 & 0 \\
\hline Infection & - & $4(9 \%)^{\ddagger}$ & 0 & 0 \\
\hline Allergic reaction & $11(23 \%)$ & 0 & 0 & 0 \\
\hline Tumor lysis syndrome & 0 & $3(6 \%)$ & $1(2 \%)$ & 0 \\
\hline Peripheral motor neuropathy & $11(23 \%)$ & $2(4 \%)$ & 0 & 0 \\
\hline Peripheral sensory neuropathy & $29(62 \%)$ & $2(4 \%)$ & 0 & 0 \\
\hline Pneumonitis & $2(4 \%)$ & $1(2 \%)$ & 0 & 0 \\
\hline Others & - & $10(21 \%)^{\S}$ & 0 & 0 \\
\hline
\end{tabular}

AST: aspartate aminotransferase;ALT: alanine aminotransferase. * Observed in $23 \%$ of all cycles of DA-EPOCH-R (Online Supplementary Table S3). 'One patient experienced tumor lysis syndrome with hyperkalemia after the first administration of rituximab. ${ }^{\prime}$ Catheter related infection $(n=1)$, cellulitis $(n=1)$, infective endocarditis ( $\left.n=1\right)$, and urinary tract infection $(n=1) .{ }^{\text {OOral mucositis }}(\mathrm{n}=3)$, hypertension $(\mathrm{n}=2)$, pulmonary embolism $(\mathrm{n}=2)$, deep vein thrombosis $(\mathrm{n}=1)$, Palmar-Planter erythrodysesthesia syndrome $(\mathrm{n}=1)$, and anorexia $(\mathrm{n}=1)$. 
that in previous reports (36-37\%). ${ }^{17,18,28}$ However, the incidence of FN per cycle of DA-EPOCH in our study was $23 \%$, which was comparable to that in previous reports for DA-EPOCH-R (19\%). ${ }^{17,40}$ The long period (7 months) of this protocol treatment may have resulted in the high proportion of patients who experienced FN. All patients with FN promptly recovered after initial antibiotic therapy and had no serious events. Hence, the toxicity could be considered manageable. Although secondary malignancy was observed in three patients, all of them were older, and two of them were treated with salvage chemotherapy after relapse. A longer follow up is needed to adequately evaluate second malignancies.

Our study has several limitations. First, it is a non-randomized phase II study with a small number of patients. Second, $96 \%$ of the patients had good PS $(<2)$. Third, the follow-up period for the evaluation of secondary malignancy was short. Fourth, considering the rarity of the disease and the feasibility of the clinical trial, this study was not designed to assess 2-year CNS relapse rate as a primary end point. Nevertheless, our results showed promis- ing efficacy and manageable toxicity of DA-EPOCHR/HD-MTX for untreated stage II-IV CD5 ${ }^{+}$DLBCL, and this trial is an important initial step in developing a more effective treatment strategy. To assess long-term efficacy and toxicity including second malignancy, a 5-year follow up is scheduled in November 2020.

\section{Acknowledgments}

We would like to thank all of the patients, hemato-oncologists, and pathologists at the participating institutes for invaluable contributions to this multicenter study.

\section{Funding}

This study was supported by grants-in-aid from the Japan Agency for Medical Research and Development, AMED (the Practical Research for Innovative Cancer Control; JP15Ack0106157, JP16ck0106157, JP17ck0106157, JP18ck0106439), the Ministry of Labour, Health, and Welfare of Japan (201438142A), the director of Mie University Hospital (2012, 2013), and the National Cancer Center Research and Development Fund (26-A-4, 29-A-3).

\section{References}

1. Swerdlow SH, Campo E, Harris NL, et al. WHO Classification of Tumours of Haematopoietic and Lymphoid Tissues. $4^{\text {th }}$ ed. Lyon: IARC Press, 2008.

2. Swerdlow SH, Campo E, Harris NL, et al. WHO Classification of Tumours of Haematopoietic and Lymphoid Tissues. Revised $4^{\text {th }}$ ed. Lyon: IARC Press, 2017.

3. Rosenwald A, Wright G, Chan WC, et al. The use of molecular profiling to predict survival after chemotherapy for diffuse large-Bcell lymphoma. N Engl J Med. 2002; 346(25):1937-1947.

4. Lenz G, Wright G, Dave SS, et al. Stromal gene signatures in large-B-cell lymphomas. N Engl J Med. 2008;359(22):2313-2323.

5. Yamaguchi $M$, Seto $M$, Okamoto $M$, et al. De novo $\mathrm{CD}^{+}$diffuse large B-cell lymphoma: a clinicopathologic study of 109 patients. Blood. 2002;99(3):815-821.

6. Yamaguchi M, Nakamura N, Suzuki R, et al. De novo $\mathrm{CD}^{+}$diffuse large B-cell lymphoma: results of a detailed clinicopathological review in 120 patients. Haematologica. 2008;93(8):1195-1202.

7. Ennishi D, Takeuchi K, Yokoyama M, et al. CD5 expression is potentially predictive of poor outcome among biomarkers in patients with diffuse large B-cell lymphoma receiving rituximab plus CHOP therapy. Ann Oncol. 2008;19(11):1921-1926.

8. Miyazaki K, Yamaguchi M, Suzuki R, et al. CD5-positive diffuse large B-cell lymphoma: a retrospective study in 337 patients treated by chemotherapy with or without rituximab. Ann Oncol. 2011;22(7):1601-1607

9. Xu-Monette ZY, Tu M, Jabbar KJ, et al. Clinical and biological significance of de novo $\mathrm{CD}^{+}$diffuse large B-cell lymphoma in Western countries. Oncotarget. 2015; 6(8):5615-5633.

10. Alinari L, Gru A, Quinion C, et al. De novo CD5+ diffuse large B-cell lymphoma: Adverse outcomes with and without stem cell transplantation in a large, multicenter, rituximab treated cohort. Am J Hematol. 2016;91(4):395-399.

11. Hyo R, Tomita N, Takeuchi K, et al. The therapeutic effect of rituximab on CD5-positive and CD5-negative diffuse large B-cell lymphoma. Hematol Oncol. 2010;28(1):2732.

12. Johnson NA, Boyle M, Bashashati A, et al. Diffuse large B-cell lymphoma: reduced CD20 expression is associated with an inferior survival. Blood. 2009;113(16):37733780

13. Salles G, de Jong D, Xie W, et al. Prognostic significance of immunohistochemical biomarkers in diffuse large B-cell lymphoma: a study from the Lunenburg Lymphoma Biomarker Consortium. Blood. 2011; 117(26):7070-7078.

14. Chuang WY, Chang H, Shih LY, et al. CD5 positivity is an independent adverse prognostic factor in elderly patients with diffuse large B cell lymphoma. Virchows Arch. 2015;467(5):571-582.

15. Suguro M, Tagawa H, Kagami $Y$, et al. Expression profiling analysis of the CD5+ diffuse large B-cell lymphoma subgroup: development of a CD5 signature. Cancer Sci. 2006;97(9):868-874.

16. Miyazaki K, Yamaguchi M, Imai $\mathrm{H}$, et al. Gene expression profiling of diffuse large BCell lymphomas supervised by CD 5 expression. Int J Hematol. 2015;102(2):188-194.

17. Wilson WH, Dunleavy K, Pittaluga S, et al. Phase II study of dose-adjusted EPOCH and rituximab in untreated diffuse large B-cell lymphoma with analysis of germinal center and post-germinal center biomarkers. J Clin Oncol. 2008:26(16):2717-2724.

18. Wilson $\mathrm{WH}$, Jung $\mathrm{SH}$, Porcu $\mathrm{P}$, et al. A Cancer and Leukemia Group B multi-center study of DA-EPOCH-rituximab in untreated diffuse large B-cell lymphoma with analysis of outcome by molecular subtype. Haematologica. 2012;97(5):758-765.

19. Grommes C, DeAngelis LM. Primary CNS Lymphoma. J Clin Oncol. 2017;35(21):24102418.

20. Miyazaki K, Yamaguchi M, Umino A, et al. DA-EPOCH-R Combined With High-dose
Methotrexate For Newly Diagnosed CD5positive Diffuse Large B-cell Lymphoma. Hematol Oncol. 2013;31:227.

21. Feugier P, Van Hoof A, Sebban C, et al. Longterm results of the R-CHOP study in the treatment of elderly patients with diffuse large B-cell lymphoma: a study by the Groupe d'Etude des Lymphomes de l'Adulte. J Clin Oncol. 2005;23(18):41174126.

22. Wilson WH, Grossbard ML, Pittaluga S, et al. Dose-adjusted EPOCH chemotherapy for untreated large B-cell lymphomas: a pharmacodynamic approach with high efficacy. Blood. 2002;99(8):2685-2693.

23. A predictive model for aggressive nonHodgkin's lymphoma. N Engl J Med. 1993; 329(14):987-994.

24. Hans CP, Weisenburger DD, Greiner TC, et al. Confirmation of the molecular classification of diffuse large B-cell lymphoma by immunohistochemistry using a tissue microarray. Blood. 2004:103(1):275-282

25 . Scott DW, Wright GW, Williams PM, et al. Determining cell-of-origin subtypes of diffuse large B-cell lymphoma using gene expression in formalin-fixed paraffinembedded tissue. Blood. 2014;123(8):1214 1217.

26. Schmitz N, Zeynalova S, Nickelsen M, et al. CNS International Prognostic Index: a risk model for CNS relapse in patients with diffuse large B-cell lymphoma treated with RCHOP. J Clin Oncol. 2016;34(26):3150-3156.

27. Cheson BD, Pfistner B, Juweid ME, et al. Revised response criteria for malignant lymphoma. J Clin Oncol. 2007;25(5):579-586.

28. Bartlett NL, Wilson WH, Jung $\mathrm{SH}$, et al Dose-Adjusted EPOCH-R Compared With $\mathrm{R}-\mathrm{CHOP}$ as Frontline Therapy for Diffuse Large B-Cell Lymphoma: Clinical Outcomes of the Phase III Intergroup Trial Alliance/CALGB 50303. J Clin Oncol. 2019; 37(21):1790-1799.

29. Thakral B, Medeiros LJ, Desai P, et al. Prognostic impact of CD5 expression in diffuse large B-cell lymphoma in patients treated with rituximab-EPOCH. Eur J Haematol. 2017;98(4):415-421 
30. Boehme V, Schmitz N, Zeynalova S, Loeffler $M$, Pfreundschuh M. CNS events in elderly patients with aggressive lymphoma treated with modern chemotherapy (CHOP-14) with or without rituximab: an analysis of patients treated in the RICOVER-60 trial of the German High-Grade Non-Hodgkin Lymphoma Study Group (DSHNHL). Blood. 2009;113(17):3896-3902

31. Villa D, Connors JM, Shenkier TN, et al. Incidence and risk factors for central nervous system relapse in patients with diffuse large B-cell lymphoma: the impact of the addition of rituximab to CHOP chemotherapy. Ann Oncol. 2010;21(5):1046-1052.

32. Gleeson M, Counsell N, Cunningham D, et al. Central nervous system relapse of diffuse large B-cell lymphoma in the rituximab era: results of the UK NCRI R-CHOP-14 versus 21 trial. Ann Oncol. 2017;28(10):2511-2516.

33. Grommes C, Pastore A, Palaskas N, et al. Ibrutinib Unmasks Critical Role of Bruton Tyrosine Kinase in Primary CNS Lymphoma.
Cancer Discov. 2017;7(9):1018-1029.

34. Grommes C, Tang SS, Wolfe J, et al. Phase $1 b$ trial of an ibrutinib-based combination therapy in recurrent/refractory CNS lymphoma. Blood. 2019;133(5):436-445.

35. Ghesquieres $\mathrm{H}$, Chevrier M, Laadhari M, et al. Lenalidomide in combination with intravenous rituximab (REVRI) in relapsed/refractory primary CNS lymphoma or primary intraocular lymphoma: a multicenter prospective 'proof of concept' phase II study of the French Oculo-Cerebral lymphoma (LOC) Network and the Lymphoma Study Association (LYSA). Ann Oncol. 2019; 30(4):621-628

36. Ferreri AJM, Calimeri T, Conte GM, et al. R$\mathrm{CHOP}$ preceded by blood-brain barrier permeabilization with engineered tumor necrosis factor-alpha in primary CNS lymphoma. Blood. 2019;134(3):252-262.

37. Klanova M, Sehn LH, Bence-Bruckler I, et al Integration of cell of origin into the clinical CNS International Prognostic Index improves CNS relapse prediction in DLBCL. Blood. 2019;133(9):919-926.

38. Vitolo U, Chiappella A, Ferreri AJ, et al. Firstline treatment for primary testicular diffuse large B-cell lymphoma with rituximab$\mathrm{CHOP}$, CNS prophylaxis, and contralateral testis irradiation: final results of an international phase II trial. J Clin Oncol. 2011;29(20):2766-2772

39. Barrans S, Crouch S, Smith A, et al Rearrangement of MYC is associated with poor prognosis in patients with diffuse large B-cell lymphoma treated in the era of rituximab. J Clin Oncol. 2010;28(20):33603365

40. Dunleavy K, Fanale MA, Abramson JS, et al. Dose-adjusted EPOCH-R (etoposide, prednisone, vincristine, cyclophosphamide, doxorubicin, and rituximab) in untreated aggressive diffuse large B-cell lymphoma with MYC rearrangement: a prospective, multicentre, single-arm phase 2 study. Lancet Haematol. 2018;5(12):e609-e617. 